\title{
Aplicación de un modelo heurístico en el diseño estratégico de la cadena de suministro: sector neumático Ecuador
}

\section{Application of a heuristic model to the strategic supply chain design: Ecuadorian rubber sector}

Jorge Campoverde

Universidad de Cuenca, Ecuador

Freddy Naula

Universidad de Cuenca, Ecuador

Denis Borenstein

Universidade Federal do Rio Grande, Brasil

Autor para correspondencia: jorgecampoverdec@gmail.com, freddybenja@gmail.com, denis.borenstein@gmail.com

Fecha de recepción: 01 de Junio de 2017 - Fecha de aceptación: 15 de Agosto de 2017

Resumen: En función del Programa Prometeo, del cual formó parte el Grupo de Investigación Empresarial (GIE) y la Carrera de Administración de Empresas de la Facultad de Ciencias Económicas y Administrativas de la Universidad de Cuenca, conjuntamente con la Dirección de Investigación de la Universidad de Cuenca (DIUC), se llevó a cabo el proyecto de Investigación "Desarrollo de Modelos Matemáticos para la Cadena de Suministro"; cuya finalidad fue implementar modelos de optimización en la Cadena de Suministro para la Industria Ecuatoriana (Sector Neumáticos). El artículo presenta una memoria respecto al desarrollo en si del proyecto, las metodologías adoptadas, dificultades en cuanto a acceso de la información, soluciones adoptadas, así como una evaluación de la Cadena de Suministro en términos generales. El aporte de ésta investigación servirá como base y marco de referencia para futuras investigaciones ya sea en la Cadena de Suministro o en Áreas Relacionadas.

Palabras clave: cadena de suministro; modelos de optimización; proyecto logístico

Abstract: According to the Prometeo Program, which included the Business Research Group (GIE) and the Business Administration Career of the Faculty of Economic and Administrative Sciences of the University of Cuenca, together with the University of Cuenca Research Directorate, the research project "Development of Mathematical Models for the Supply Chain" was carried out; Whose purpose was to implement optimization models in the Supply Chain for the Cuencan industry (Tires Sector). This article presents a report on the development of the project itself, the methodologies adopted, the difficulty of obtaining information, adopted solutions, as well as an evaluation of the Supply Chain in general terms; All serve as a frame of reference for future research in either the Supply Chain or Related Areas.

Key words: supply chain; optimization models; logistic project 


\section{Introducción}

La cadena de suministro (CS) se la puede considerar como un proceso integrado en el cual varios negocios (i.e., proveedores, productores, distribuidores, y detallistas) trabajan juntos con la finalidad de adquirir materias primas, convertir éstas en productos, y entregarlos al cliente final (Beamon, 1998). La CS se caracteriza generalmente por mantener un flujo de materiales y/o productos hacia adelante, y de información en ambas direcciones. Las empresas se motivan a ser parte de esta compleja red, debido a las recompensas monetarias (David Simchi-Levi, Xin Chen, 2004), cuyo impacto en la economía global es hoy más relevante que en ningún otro momento; en este sentido (David Simchi-Levi, Xin Chen, 2004) consideran que el paso más importante para reducir los costos en la cadena de suministro es el Diseño de la Red de la Cadena de Suministro (DRCS), por lo tanto el DRCS, involucra la toma de decisiones tanto a nivel estratégico i.e., número de instalaciones, localizaciones, capacidades y tecnología de las mismas; como a nivel táctico u operativo i.e., cantidades agregadas, flujos de las compras, procesamiento y distribución de los productos (Santoso, Ahmed, Goetschalckx, \& Shapiro, 2005); el número de etapas se determina de acuerdo a la complejidad de los problemas fragmentados en la red (Pirkul \& Jayaraman, 1998), además siempre es importante desarrollar competencias a lo largo de la red, para mejorar la competitividad de las empresas (Rice \& Hoppe, 2001). Por lo tanto, el DRCS, debe considerar necesariamente las capacidades de cada uno de los elementos que conforman la red, pues son aspectos importantes para la sostenibilidad y competitividad de una empresa (Zhang, Lee, \& Zhang, 2016).

Las técnicas de investigación operativa están siendo cada vez más utilizadas para la resolución del problema de DRCS, con el afán de proporcionar una solución no solo factible sino en muchos de los casos óptimos. La modelación matemática generalmente busca minimizar los costos de mover los materiales a lo largo de la red, sin que por ello se comprometan los niveles de servicio ofrecidos al cliente (Farias \& Denis, 2017).

En este estudio se presentan las experiencias en el desarrollo e implementación de un modelo de Programación Lineal Entera Mixta aplicado a una empresa importante de la ciudad de Cuenca, cuyo giro del negocio se basa principalmente en la producción de Neumáticos, misma que cuenta con zonas de consumo definidas, así como con proveedores y distribuidores.

El proyecto es el resultado de la fusión de dos auspicios: el primero, el Proyecto Prometeo para docentes e investigadores en Ecuador, impulsado por el Gobierno Nacional; y el segundo, la Dirección de Investigación de la Universidad de Cuenca (DIUC), en función de lo mencionado se ha trabajado este artículo, el cual se estructura de la siguiente forma: en la sección 1 realizamos una introducción, posteriormente en la sección 2 se presenta la metodología utilizada, en la cual se incluye también el modelo matemático, algunas fórmulas utilizadas para el cálculo de los costos logísticos y el diseño de un sistema de Información para la CS; en la sección 3 se contextualiza y finalmente se presentan las conclusiones.

\section{Metodología}

\section{Modelo Matemático}


La compañía oferta al mercado nacional e internacional un conjunto de productos elaborados de caucho; los mismos que pueden ser de diferentes tamaños y características. Las plantas de producción reciben materias primas de varios proveedores; el conjunto de Centros de Distribución (CD), recibe una amplia gama de productos de las diferentes plantas de producción, paralelamente los CD son responsables de enviar cantidades establecidas de productos a las diferentes zonas de consumo, de acuerdo a la demanda establecida; de esta manera se completa la Red de la Cadena de Suministro, la misma que está conformada por los proveedores de materias primas, plantas de producción, centros de distribución y zonas de consumo.

En la cadena de suministro se genera la necesidad de movilizar productos, por lo tanto es importante conocer los costos de transportación de las materias primas y de los distintos productos en cada fase de la cadena, así como también se debe considerar la capacidad de producción de cada planta, y las de procesamiento en los Centros de Distribución. Consecuentemente es necesario considerar costos fijos y variables.

El modelo matemático desarrollado, se basó en la formulación presentada por (Pirkul \& Jayaraman, 1998), en el cual las variables continuas están definidas en función de las cantidades de materia prima que suministran los proveedores a las plantas, y las cantidades de productos (variables enteras) que fluyen desde las fábricas hacia las zonas de consumo a través de los distintos Centros de Distribución.

El problema básicamente consiste en determinar: el conjunto de CDs necesarios para satisfacer la demanda de productos en las diferentes Zonas de Consumo (ZC) con el costo operacional más bajo, la cantidad de materia prima a solicitar a cada proveedor, así como también determinar las cantidades a producir en las diferentes plantas.

Una zona de consumo puede ser atendida exclusivamente por un CD, lo cual es conocido como modelo de "Distribuidor Único", en el caso en el cual una determinada zona de consumo sea atendida por más de un CD, lo llamaremos modelo de "Múltiple Distribuidor", a continuación definiremos algunas variables a utilizar:

C: Conjunto de zonas de consumo (Clientes), asignados como c;

W: Conjunto de CDs, (almacenes), asignados como w;

F: Conjunto de plantas de fabricación; asignados como f;

R: Conjunto de materias primas; asignados como r;

$\mathrm{V}$ : Conjunto de proveedores; asignados como v;

$\mathrm{S}$ : Conjunto de productos; asignados como s;

P: Conjunto de rutas; asignadas como $\mathrm{p}$.

Parámetros Utilizados:

$d_{s c}$ : Demanda para el producto $s \in S$ de la zona de consumo $c \in C$;

$U_{f}$ : Número máximo de plantas que pueden ser abiertas;

$U_{w}$ : Número máximo de centros de distribución que pueden ser abiertos;

$u_{r s}$ : Porcentaje de uso de materia prima $r \in R$ para el producto $s \in S$;

$u_{s}$ : Porcentaje de la capacidad de uso para el producto $s \in S$;

$C A P_{W}$ : Capacidad de transferencia de los centros de distribución;

$C A P_{v r}$ : Capacidad de proveer de materia prima $r \in R$ por el proveedor $v \in V$; 
$C A P_{f}$ : Capacidad de producción de la planta $f \in F$;

$C T_{f}^{o}$ : Costos fijos anuales para el funcionamiento de la planta $f \in F$;

$C T_{w}^{o}$ : Costos fijos anuales para el funcionamiento del centro de distribución $w \in W$;

$C T_{w}^{g}$ : Costos unitarios de transferencia para el funcionamiento del centro de distribución $w \in$ $W$;

$C T_{f s}^{p}$ : Costo unitario de producción para el producto $s \in S$ en la planta $f \in F$;

$C T_{f v r}^{t}$ : Costo unitario de transporte de materia prima $r \in R$ desde el proveedor $v \in V$ para la planta $f \in F$;

$C T_{p s}^{t}$ : Costo unitario de transporte del producto $s \in S$ usando la vía $p \in P$;

$C T_{f w s}^{t}$ : Costo unitario de transporte del producto $s \in S$ usando la planta $f \in F$ para el centro de distribución $w \in W$;

$C T_{w C S}^{t}$ : Costo unitario de transporte del producto $s \in S$ usando el centro de distribución $w \in W$ a la zona de consumo $c \in C$;

$u_{\text {min }}$ : Demanda mínima para abrir un Centro de Distribución $w \in W$;

\section{Modelo Distribuidor Único}

La figura 1 ejemplifica el concepto de Distribuidor Único, demostrando que una zona de consumo puede ser atendida por exclusivamente un centro de distribución.

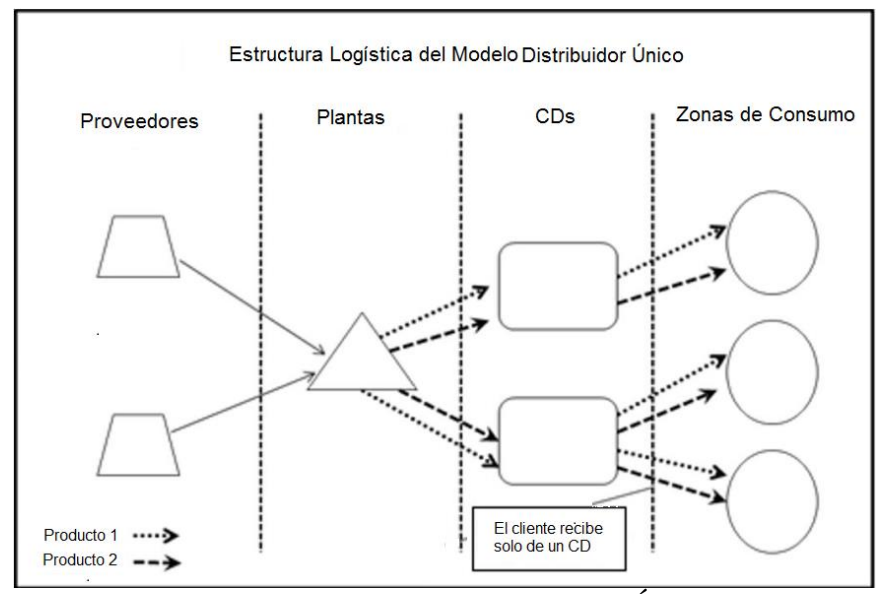

Figura 1 Modelo de Distribuidor Único

Se exponen a continuación las siguientes variables, de acuerdo al presente modelo:

$x_{f w s}$ : Cantidad del producto $s \in S$, enviado desde la planta $f \in F$, al centro de distribución $w \in$ $W$

$y_{v f r}$ : Cantidad de la materia prima $r \in R$, enviado desde el proveedor $v \in V$, al centro de distribución $f \in F$;

$a_{w}$ : Variable binaria que asume el valor 1 si el centro de distribución $w \in W$ es seleccionado, caso contrario 0 ;

$g_{w c}$ : Variable binaria que indica si el centro de distribución $w \in W$ satisface la demanda de la zona de consumo $c \in C$; 
Una vez que se han definido las variables, el problema puede ser considerado como de Programación Lineal Entera Mixta, como se indica a continuación:

\section{Formulación Matemática}

$$
\begin{gathered}
\min \left(\sum_{w \in W} C T_{w}^{o} a_{w}+\sum_{w \in W} \sum_{c \in C} \sum_{s \in S} C T_{w}^{g} d_{s c} g_{w c}+\sum_{f \in F} \sum_{w \in W} \sum_{s \in S} C T_{f S}^{p} x_{f w s}+\right. \\
\left.\sum_{v \in V} \sum_{f \in F} \sum_{r \in R} C T_{f v r}^{t} y_{v f r}+\sum_{f \in F} \sum_{w \in W} \sum_{s \in S} C T_{f w s}^{t} x_{f w s}+\sum_{w \in W} \sum_{c \in C} \sum_{s \in S} C T_{w c S}^{t} d_{s c} g_{w c}\right)
\end{gathered}
$$

(1a)

$$
\begin{array}{ll}
\text { St: } & \\
\sum_{w \in W} g_{w c}=1 & \forall c \in C \\
\sum_{c \in C} \sum_{s \in S} d_{s c} g_{w c} \leq C A P_{w} z_{w} & \forall w \in W \\
\sum_{w \in W} a_{w} \leq U_{w} & \\
\sum_{c \in C} d_{s c} g_{w c} \leq \sum_{f \in F} x_{f w s} & \forall w \in W, \forall c \in C \\
\sum_{f \in F} y_{v f r} g_{w c} \leq C A P_{v r} & \forall r \in R, \forall v \in V \\
\sum_{w \in W} \sum_{s \in S} u_{r s} x_{f w s} \leq \sum_{v \in V} y_{v f r} & \forall r \in R, \forall f \in F \\
\sum_{w \in W} \sum_{s \in S} u_{s} x_{f w s} \leq C A P_{f} & \forall f \in F \\
\sum_{f \in F} \sum_{s \in S} x_{f w s} \geq u_{\min } & \forall w \in W \\
a_{w}=\{0,1\} & \forall w \in W \\
g_{w c}=\{0,1\} & \forall w \in W, \forall c \in C
\end{array}
$$

La función objetivo minimiza la suma de Costos fijos anuales del funcionamiento de los CDs, Costo unitario de transferencia para el funcionamiento de los centros de distribución, Costo unitario de producción para el producto, Costo unitario de transporte de la materia prima desde el proveedor a la planta, Costo unitario de transporte de producto de la planta al CD y Costo unitario de transporte de producto del CD a la ZC. La restricción (1b) asegura que una zona de consumo es atendida por un CD, bajo el enfoque de "Distribuidor Único". Por su lado la restricción (1c) garantiza que la capacidad de cada centro de distribución sea respetada. La restricción (1d) limita el número de CD a ser abiertos. Mediante la restricción (1e) se asegura que los CD tengan suficiente capacidad para atender a las zonas de consumo. La restricción (1f) garantiza la capacidad de entrega de los proveedores a la planta. En la restricción (1h) se asegura que las capacidades de las plantas sean respetadas. A su vez la restricción (1i) permite imponer un mínimo de productos necesarios para abrir un centro de distribución. Las restricciones (1j) y (1k) son condiciones de integridad de las variables binarias.

\section{- Modelo Múltiple Distribuidor}

El Diseño de la Red de la Cadena de Suministro (DRCS), denominado Múltiple Distribuidor, es un modelo que propone usar líneas de abastecimiento entre las plantas, los CD y las Zonas de Consumo. Las variables de decisión están definidas en las líneas de abastecimiento, representando los flujos de productos y de materias primas entre elementos en dos niveles consecutivos de la CS. Ésta formulación se la denomina "una a muchas", porque el modelo permite a un cliente recibir productos de más de un $\mathrm{CD}$, también considera una estructura que limita la cantidad de plantas que se pueden abrir. El modelo se presenta en la figura 2. 


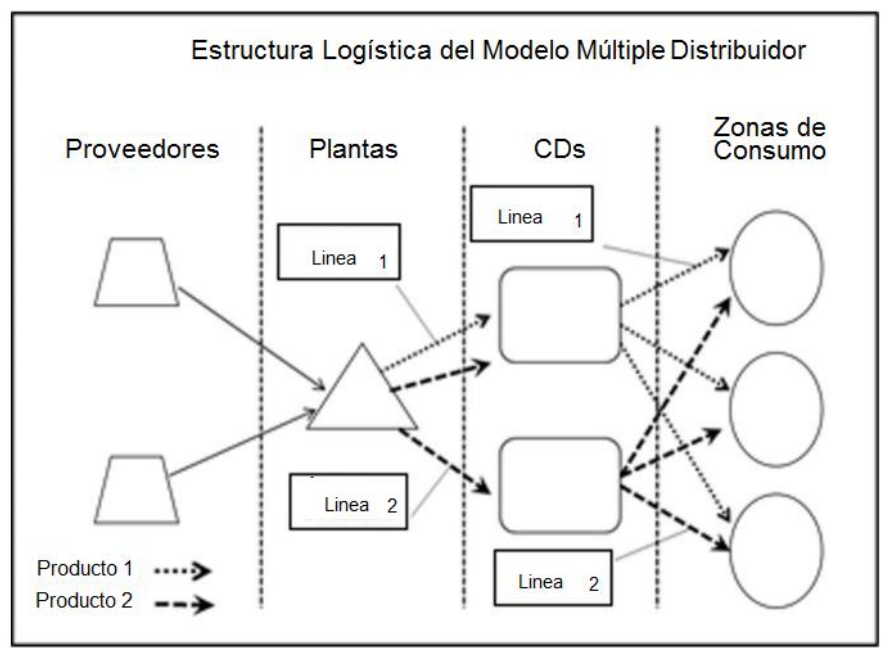

Figura 2 Modelo Múltiple Distribuidor

El modelo de Múltiple Distribuidor requiere de los costos de transporte ya definidos entre las plantas, CD, y Zonas de Consumo. Se definen las siguientes variables:

$x_{w c}^{S}$ : Cantidad del producto $s \in S$ enviado desde los centros de distribución $w \in W$ a las zonas de consumo $c \in C$;

$y_{v f}^{r}$ : Cantidad de la materia prima $r \in R$ enviados desde el proveedor $v \in V$ a la planta $f \in F$; $z_{f w}^{S}$ : Cantidad del producto $s \in S$ enviado desde la planta $f \in F$ al centro de distribución $w \in$ $W$

$a_{w}$ : Variable binaria que asume el valor 1 si el centro de distribución $w \in W$ es seleccionado, caso contrario 0 ;

$b_{f}$ : Variable binaria que asume el valor 1 si la planta $f \in F$ es seleccionado, caso contrario 0 ;

El problema puede ser formulado de la siguiente manera:

$$
\begin{aligned}
& \min \left(\sum_{w \in W} C T_{w}^{o} a_{w}+\sum_{w \in W} C T_{f}^{o} b_{f}+\sum_{f \in F} \sum_{s \in S} \sum_{w \in W} C T_{f s}^{p} z_{f w}^{s}+\sum_{w \in W} \sum_{c \in C} \sum_{s \in S} C T_{w}^{g} x_{w c}^{s}+\right. \\
& \left.\sum_{f \in F} \sum_{r \in R} \sum_{v \in V} C T_{f v r}^{t} y_{v f}^{r}+\sum_{f \in F} \sum_{w \in W} \sum_{s \in S} C T_{f w s}^{t} z_{f w}^{s}+\sum_{w \in W} \sum_{c \in C} \sum_{s \in S} C T_{w c S}^{t} x_{w c}^{S}\right) \\
& \text { 2(a) } \\
& \text { St: } \\
& \sum_{w \in W} x_{w c}^{S} \geq d_{c}^{S} \\
& \sum_{c \in C} x_{w c}^{s}=\sum_{f \in F} z_{f w}^{S} \\
& \forall s \in S, \forall c \in C \\
& \forall s \in S, \forall w \in W \\
& \sum_{c \in C} \sum_{s \in S} x_{w c}^{S} \leq C A P_{w} a_{w} \\
& \sum_{f \in F} y_{v f}^{r} \leq C A P_{v r} \\
& \forall w \in W \\
& \forall r \in R ; \forall v \in V \\
& \sum_{w \in W} \sum_{s \in S} u_{r s} z_{f w}^{s} \leq \sum_{v \in V} y_{v f}^{r} \quad \forall r \in R ; \forall f \in F \\
& \sum_{w \in W} \sum_{s \in S} u_{r s} z_{f w}^{s} \leq C A P_{f} b_{f} \quad \forall f \in F \\
& \sum_{w \in W} a_{w} \leq U_{w} \\
& \sum_{f \in F} b_{f} \leq U_{f} \\
& a_{w}=\{0,1\} \\
& \forall w \in W \\
& b_{f}=\{0,1\} \quad \forall f \in F
\end{aligned}
$$


La función objetivo (2a) minimiza la suma de: Costo fijo anual del funcionamiento del $\mathrm{CD}$, Costos fijos anuales para el funcionamiento de la planta, Costo unitario de producción, Costo unitario de transferencia para el funcionamiento del CD, Costo unitario de transporte de materia prima del proveedor a la planta, Costo unitario de transporte del producto desde la planta al CD y el Costo unitario de transporte de producto del CD a la ZC. La restricción (2d) asegura que la capacidad de cada CD sea respetada, La restricción (2e) segura la capacidad de los proveedores en la entrega de materias primas, La restricción (2f) garantiza la relación entre cantidad de materia prima y cantidad de producto, la restricción ( $2 \mathrm{~g}$ ) asegura que la capacidad de las plantas se cumpla, las restricciones $(2 \mathrm{j})$ y $(2 \mathrm{k})$ son condiciones de integridad de las variables binarias.

Referencias para Calcular Costos. En la cadena de suministro, se involucran también otras actividades, como por ejemplo: manejo de inventario, almacenamiento, producción, distribución, etc. (Arshinder, Kanda, \& Deshmukh, 2008); sin embargo lo más común es que se defina como un sistema de proveedores, productores, distribuidores, detallistas y clientes; en donde fluyen los materiales en una sola dirección (desde los productores a los clientes) y la información en ambas direcciones (Houlihan, 1987; Lamming, 1996; Lee \& Billington, 1993; Stevens, 1989). Como se mencionó anteriormente, es necesario obtener los costos de aprovisionamiento, almacenamiento y distribución, por lo tanto, es importante determinar o identificar los costos involucrados en el flete en cada caso $(\$ / \mathrm{Km})$, los mismos que se obtienen de la siguiente fórmula:

En dónde;

$$
\begin{aligned}
C F k m & =\frac{(C F t)(C t p)}{R m} \\
C F t & =\left(1+\frac{G a}{100}\right)(C f t+C v t+C i t) \\
C f t & =\frac{\sum_{n=1}^{N} C f_{n}}{(v)(C t p)} \\
C v t & =\left(\frac{R m}{C t p}\right)\left(\sum_{n=1}^{N} C v_{n}\right) \\
C i t & =\frac{\sum_{n=1}^{N} C i_{n}}{t m}
\end{aligned}
$$

CFkm: Costo del flete por Kilómetro

$C F t$ : Costo del Flete por Tonelada

Ctp: Capacidad de tonelaje promedio del vehículo.

$R m$ : Recorrido mensual

Ga: Ganancia o rentabilidad

Cft: Costos fijos por tonelada

Cvt: Costos variables por tonelada

Cit: Costos indirectos por tonelada

$C f_{n}$ : Costo fijo n.

$C v_{n}$ : Costo variable $\mathrm{n}$.

$V$ : Número de viajes mensuales.

$C i_{n}$ : Costo indirecto $\mathrm{n}$. 
Tm: Tonelaje mensual expedido

En cuanto al cálculo de costos de almacenamiento y distribución, para los distintos productos se observó la utilización de un índice logístico (Lambán, Royo, Valencia, Berges, \& Galar, 2013), la fórmula de repartición para los productos fue modificada y se adaptó al contexto.

$$
\begin{array}{ll}
I_{i}=\propto * I p_{i}+\beta * I v_{i} & 4(a) \\
I p_{i}=\frac{P_{i}}{\sum_{n=1}^{N} P_{n}} & 4(b) \\
I p_{i}=\frac{V_{i}}{\sum_{n=1}^{N} V_{n}} & 4(c)
\end{array}
$$

En donde:

$I_{i}$ : Indice logistico del artículo i

$I p_{i}$ : Indice del peso del artículo i

$I v_{i}:$ Indice del volumen del artículo i

$P_{i}$ : Peso del artículo i

$V_{i}$ : Volumen del artícuo i

$$
\begin{aligned}
& \propto+\beta=1 \\
& (\alpha, \beta) \in N
\end{aligned}
$$

Tanto Alfa como Beta toman un valor de 0.5, sin embargo, éstos pueden variar de acuerdo al grado de importancia que se le otorgue al peso y/o al volumen con respecto al producto transportado y su influencia en el furgón o camión.

La fórmula para la repartición de costos directos de almacenamiento (5), ya modificada está dada por:

$$
\operatorname{Cmod}_{i}=\sum_{k=1}^{K}\left(\frac{\left(\operatorname{Cmod}_{k}\right) * I_{i} * M A_{i, k}}{\sum_{n=1}^{N}\left(I_{n} * M A_{n, K}\right)}\right)
$$

En donde:

$C \bmod A_{i}$ : Costo de mano de obra directa del articulo $i$

$\mathrm{Cmod}_{k}$ : Costo de mano de obra del empleado $\mathrm{k}$

$M A_{i ; k}$ : Numero de manipulaciones del articulo $i(n)$ por parte del empleado $k$

Similar operación se realiza para costos de infraestructura y el resto de costos directos realizando las adaptaciones que correspondan.

\section{Sistema de Información}

El desempeño de la cadena de suministro es un factor determinante para el éxito de la empresa (Nunes, Annansingh, \& Eaglestone, 2006). Además, considerando el desarrollo de la 
economía hacia un entorno cada vez más globalizado, es necesario crear, adquirir y diseminar información y conocimiento apropiado. Para que la empresa pueda responder de mejor manera a las exigencias del cliente y a los cambios del mercado es necesario que comparta la información dentro de sí, lo cual incluye también a la CS (Lotfi, Mukhtar, Sahran, \& Zadeh, 2013).

En este contexto, la organización debe responder básicamente a cuatro preguntas: ¿qué compartir?, ¿con quién compartir?, ¿cómo compartir?, y ¿cuándo compartir?; en base a la calidad de las respuestas a las preguntas planteadas se podrán: evitar redundancias, reducir costos y mejorar resultados (Pérez-Méndez \& Machado-Cabezas, 2015; Sun \& Yen, 2005); Todo lo anterior servirá además como insumo importante para el diseño de estrategias administrativas (Martinez-Simarro, Devece, \& Llopis-Albert, 2015). Hay que considerar sin embargo que el entorno dinámico y cada vez más complejo, obliga a la empresa a utilizar nuevos y mejores sistemas de información (El-Telbany \& Elragal, 2014) con la finalidad de que la información sea consistente y oportuna (Doherty \& Terry, 2009), estos sistemas han de involucrar en mayor medida posible a todos los actores de la cadena de suministro.

En este trabajo se introducen particularidades que permiten que el sistema se adecue y responda de la mejor manera posible. En la figura 3 se presenta el diagrama relacional, diseñado para el levantamiento de información base a ser utilizada en el proyecto; El sistema de información fue implementado en MySql WorkBench. 


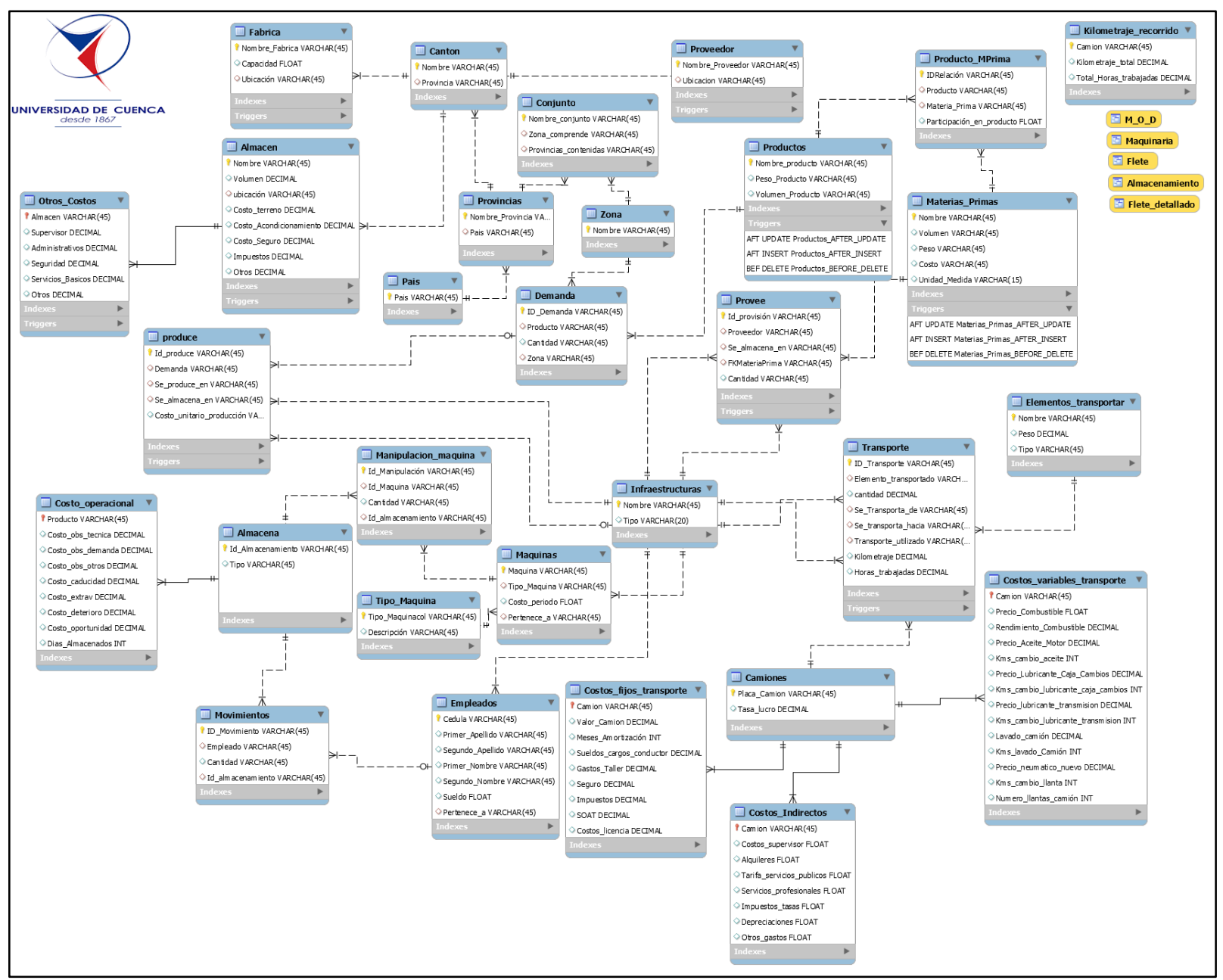

Figura 3 Modelo Relacional del SI. Generado en MYSql.

\section{Contextualización:}

En base al modelo ejecutado, lo más conveniente para la industria de los neumáticos del país es proveer el producto segmentando el mercado en básicamente tres zonas de consumo: Norte, Oeste y Sur. La zona Norte comprende a Pichincha y sus provincias vecinas incluidas algunas del oriente. La zona Sur abarca a Azuay y a las provincias circundantes, incluyendo cierta parte del oriente. Finalmente la zona Oeste está comprendida por Guayas y las provincias que la rodean. 


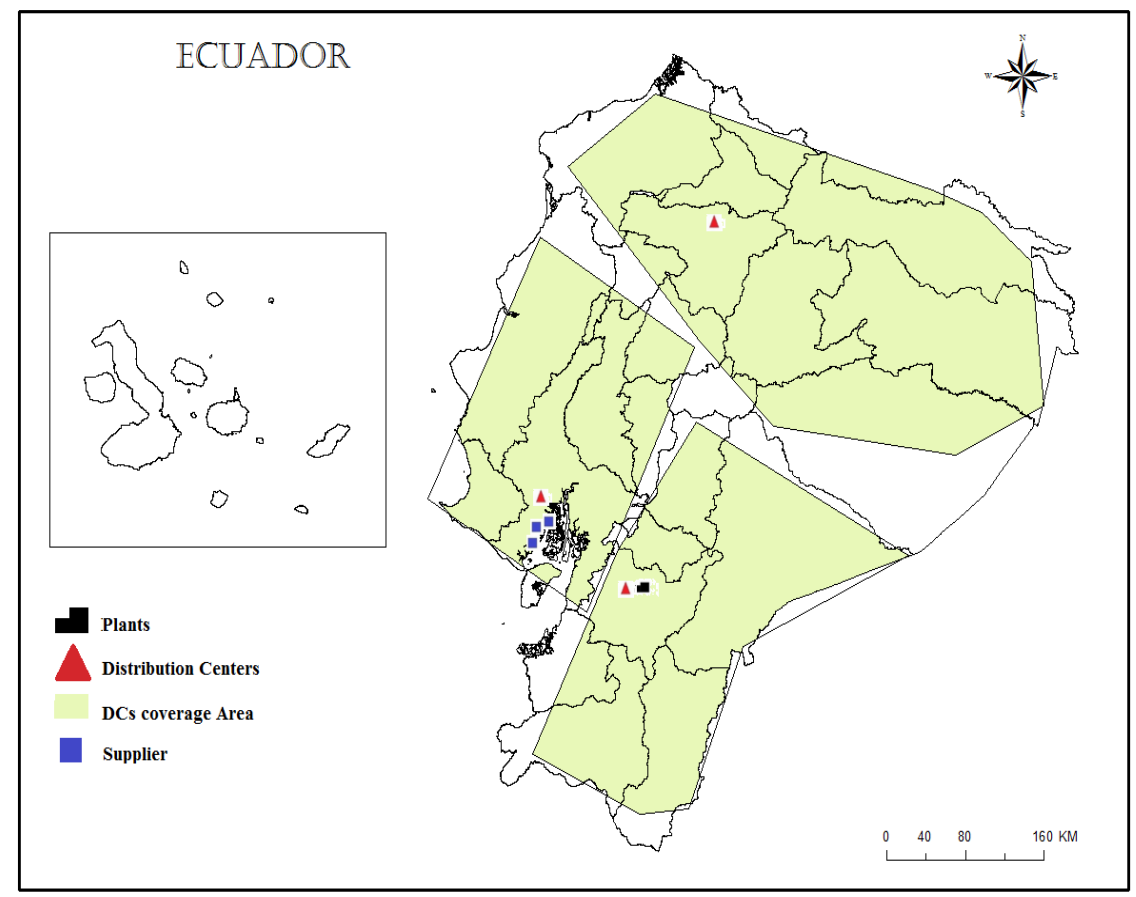

Figura 4 Zonas de Consumo.

El grado de dificultad en la ejecución del presente modelo, puede ser mucho más complejo en el caso de países, o zonas geográficamente extensas. Dado el tamaño del mercado nacional es suficiente una sola planta de producción. Es necesario acotar que la ubicación de los CDs nacionales, que proveen de inventario a las zonas de consumo coincide con las principales ciudades del país, consideradas como polos de desarrollo regionales. En general, los parámetros base son: 35 tipos de productos, 8 zonas de consumo ( 3 nacionales y 5 extranjeras), 3 Centros de Distribución, 7 materias primas fundamentales, 17 proveedores y una sola planta de producción. Para la implementación del modelo se utilizó el software IBM ILOG CPLEX.

En la fase de levantamiento de datos, se comprobó que no existe información a un nivel de detalle adecuado para el modelo. Se observó que la empresa básicamente distribuye su producción en dos tipos de mercados: el de repuesto y el original. El mercado de repuesto está compuesto básicamente por las ventas al detalle en el país; y el original por las ensambladoras nacionales de automóviles.

Una implementación 100\% efectiva requiere contar con la máxima colaboración de la empresa, en cuanto a suministro de información; éste no es el caso, ya que el empresario ecuatoriano normalmente no proporciona toda la información, pues considera que es parte del giro de su negocio (know-How), ésta situación si bien no merma validez al modelo, si exactitud.

\section{Conclusiones}

Los modelos desarrollados "Distribuidor Único" y "Múltiple Distribuidor" representan esquemas factibles para la optimización de la cadena de Suministro; sin embargo dadas las circunstancias propias de cada una de las industrias; en el presente caso, el modelo más adecuado resulta ser el de "Distribuidor Único". 
Con el modelo "Distribuidor Único" se obtiene una relación cliente proveedor más personalizada; sin embargo los costos mantienen una ligera desventaja frente al Modelo "Múltiple Distribuidor", ya que las opciones de aprovisionamiento existentes se restringen.

En última instancia, la elección del modelo más adecuado será responsabilidad de la alta gerencia, quienes tendrán que sopesar entre personalización en el aprovisionamiento o tener costos menores.

Uno de los objetivos principales de este estudio fue establecer el número de CDs que han de formar parte de la CS, con los modelos desarrollados se logran cubrir de manera íntegra la cadena de suministro. Se determinó qué proveedores deben entregar a la planta sus productos, que cantidad de materias primas, considerando la demanda del producto en cada zona de consumo. Ambos modelos fueron implementados de tal manera que las soluciones obtenidas logren cubrir totalmente las demandas requeridas; con valores de acuerdo al propósito de minimización de costos operacionales.

\section{Bibliografía}

Arshinder, Kanda, A., \& Deshmukh, S. G. (2008). Supply chain coordination: Perspectives, empirical studies and research directions. International Journal of Production Economics, 115(2), 316-335. http://doi.org/10.1016/j.ijpe.2008.05.011

Beamon, B. M. (1998). Supply chain design and analysis: Models and methods. International Journal of Production Economics, 55(3), 281-294. http://doi.org/10.1016/S09255273(98)00079-6

David Simchi-Levi, Xin Chen, J. B. (2004). The Logic of Logistics: Theory, Algorithms, and Applications for Logistics and Supply Chain Management. Springer.

Doherty, N. F., \& Terry, M. (2009). The role of IS capabilities in delivering sustainable improvements to competitive positioning. Journal of Strategic Information Systems, 18(2), 100-116. http://doi.org/10.1016/j.jsis.2009.05.002

El-Telbany, O., \& Elragal, A. (2014). Business-information Systems Strategies: A Focus on Misalignment. $\quad$ Procedia 250-262. http://doi.org/10.1016/j.protcy.2014.10.090

Farias, E. da S., \& Denis, B. (2017). Modeling the logistics design of a multi-commodity industry. Gestión Y Producción, 148-160.

Houlihan, J. B. (1987). International Journal of Physical Distribution \& Logistics Management International Supply Chain Management.

Lambán, M. P., Royo, J., Valencia, J., Berges, L., \& Galar, D. (2013). Almacenamiento para el cáluculo del costo de almacenamiento de un producto: Caso de estudio en un entorno 
logístico. Dyna - Medellín, (179), 23-32. Retrieved from http://www.scielo.org.co/pdf/dyna/v80n179/v80n179a03.pdf

Lamming, R. (1996). Squaring lean supply with supply chain management. International Journal of Operations \& Production Management, 16(2), 183-196. http://doi.org/10.1108/01443579610109910

Lee, H. L., \& Billington, C. (1993). Material management in decentralized supply chains. Operations Research, 41(5), 835. http://doi.org/10.1287/opre.41.5.835

Lotfi, Z., Mukhtar, M., Sahran, S., \& Zadeh, A. T. (2013). Information Sharing in Supply Chain Management. Procedia Technology, 11(Iceei), 298-304. http://doi.org/10.1016/j.protcy.2013.12.194

Martinez-Simarro, D., Devece, C., \& Llopis-Albert, C. (2015). How information systems strategy moderates the relationship between business strategy and performance. Journal of Business Research, 68(7), 1592-1594. http://doi.org/10.1016/j.jbusres.2015.01.057

Pérez-Méndez, J. A., \& Machado-Cabezas, Á. (2015). Relationship between management information systems and corporate performance. Revista de Contabilidad, 18(1), 32-43. http://doi.org/10.1016/j.rcsar.2014.02.001

Pirkul, H., \& Jayaraman, V. (1998). A multi-commodity, multi-plant, capacitated facility location problem: formulation and efficient heuristic solution. Computers \& Operations Research, 25(10), 869-878. http://doi.org/10.1016/S0305-0548(97)00096-8

Rice, J. B., \& Hoppe, R. M. (2001). Supply chain vs supply chain. The hype \& the reality. Supply Chain Management Review, (September/october), 46-54.

Santoso, T., Ahmed, S., Goetschalckx, M., \& Shapiro, A. (2005). A stochastic programming approach for supply chain network design under uncertainty. European Journal of Operational Research, 167(1), 96-115. http://doi.org/10.1016/j.ejor.2004.01.046

Stevens, G. C. (1989). Integrating the Supply Chain. International Journal of Physical Distribution \& Materials Management, 19(8), 3-8. http://doi.org/10.1108/EUM0000000000329

Sun, S., \& Yen, J. (2005). Information Supply Chain: A unified framework for informationshraing. Intelligence and Security Informatics, 3495, 422-428.

Zhang, L. L., Lee, C., \& Zhang, S. (2016). An integrated model for strategic supply chain design: Formulation and ABC-based solution approach. Expert Systems with Applications, 52, 3949. http://doi.org/10.1016/j.eswa.2015.12.035 